\title{
1 Enhancing Robusta Coffee aroma by modifying flavour precursors in the green
}

3

4

5

6

7

\section{Keywords:}

9 Coffee processing; Green bean pre-treatment; Sugar; Shelf-life; Sensory analysis; Aroma chemistry

10 Highlights:

11 1. Varying levels of sugars were used to pre-treat Robusta green beans.

12 2. Treatment increased the similarity of Robusta to Arabica.

13 3. The optimum level of sugar treatment was Robusta soaked in $15 \mathrm{~F}$ solution.

14 4. For coffee aroma the blending ratio can be increased from $20 \%$ to $80 \%$ Robusta.

15 5. The aroma of treated Robusta coffee was more stable than Arabica. 


\section{Abstract}

17 This study attempted to improve Robusta sensory properties by modifying the beans chemical 18 composition. Building on our previous work, which modified bean $\mathrm{pH}$ through acid pre-treatment, a 19 model system was developed where, sugar solutions (glucose, fructose, sucrose) were used to pre-treat 20 Robusta coffee beans with the aim to modify the concentration/availability/location of these aroma 21 precursors. Beans were then dried to equal water activity, subjected to equal roast intensity and ground 22 to comparable particle size distributions. The treatment significantly impacted aroma generation during 23 roasting leading to an altered level of pyrazines, furans, ketones, organic acid and heterocyclic nitrogen24 containing compounds $(\mathrm{p}<0.05)$. The optimum treatment was $15 \mathrm{~g} / 100 \mathrm{~g}$ fructose. $80 \%$ treated Robusta 25 could be blended with Arabica in coffee brew without significant aroma differences being perceived 26 when compared to $100 \%$ Arabica brew. Furthermore the aroma of the fructose treated Robusta was more 27 stable than Arabica over six weeks accelerated shelflife storage. 
Being a popular beverage worldwide, coffee demand and consumption have increased significantly over recent years. The International Coffee Organization estimated that two billion cups are consumed every day and of which the fastest growing segment is for premium coffee, therefore there is an urgent need to improve beverage quality without increasing cost (International Coffee Organization, 2016). Cup quality depends on various factors therefore scientists have found it challenging to improve coffee quality due to the complexity within the bean and the processing.

Green coffee bean chemical composition plays an important role in aroma formation during the roasting process (Fisk, Kettle, Hofmeister, Virdie, \& Kenny, 2012). The Maillard reaction is the major pathway of aroma formation in coffee, amino acids and reducing sugars react to form nitrogenous heterocycles and brown melanoidins (Illy \& Viani, 2005). This non-enzymatic browning produces hundreds of volatile compounds, and contributes to a number of sensory attributes of coffee (Lersch, 2012). Controlling the precursors (sugars, amino acids) and the process will therefore enable control over the aroma generation and the final flavour of the coffee (Wong, Abdul Aziz, \& Mohamed, 2008).

The two main cultivated species of coffee are Arabica (Coffeea Arabica L.) and Robusta (Coffeea canephora P.) (Illy \& Viani, 2005). Previous studies have showed that Arabica has a sweet, caramel roast aroma whilst Robusta has an earthy, spicy roast aroma (Blank, Sen, \& Grosch, 1991). Sucrose is considered important for the development of the organoleptic qualities of coffee and Robusta has significantly less $(2.7 \%$ dry weight) compared to the $6 \%(\mathrm{dwb})$ that is found in Arabica (Illy \& Viani, 2005). The higher sucrose content results in an enhanced aroma formation for Arabica (Farah, 2012). In Argentina, Spain and Singapore, there is a special type of roasted coffee called Torrefacto which it is 
sugar during roasting process) (Wrigley, 1988). The sugar added in this treatment is proposed not to increase the sweetness of the coffee brew but to protect the beans from oxidation by forming a thin sugar film on the surface and to speed up the Maillard reaction (Wrigley, 1988). This procedure has also been demonstrated to mask the poor quality of low grade beans, especially Robusta (Lersch, 2012).

Our previous study involved the treatment of green coffee beans with a solution containing varying concentration of acetic acid for $2 \mathrm{~h}$ at $20^{\circ} \mathrm{C}$, with the aim to change the acidity of bean prior to roasting therefore diverting the kinetics of certain reaction pathways that occur during aroma formation during roasting, this treatment reduced the aroma differences between Arabica and Robusta and enabled a higher blending ratio (Liu, Yang, Linforth, Fisk, \& Yang, 2018). We are building on this previous work, that highlighted the importance of the local microchemistry $(\mathrm{pH})$ on aroma generation, and offer an alternative, more targeted method to alter the concentration/availability/location of sugar precursors for Maillard chemistry and caramelisation reactions that occur during roasting. Instead of modifying the local solvent micro-chemistry $(\mathrm{pH})$, the objective of this study is therefore to develop a model system that allows us for the first time to individually modify the green bean chemical precursors (sucrose, glucose and fructose), and individually evaluate their impact on the coffee aroma generation and to show that modification of flavour precursors could be used to increase the aroma similarity between Arabica and Robusta coffee and further to understand the impact on aroma stability over shelf life.

Compared with Torrefacto process, instead of adding sugar during the roasting process, our study modified the flavour precursors content in the green beans prior to roasting. Green Robusta beans were pre-soaked in solutions of both reducing sugars (glucose and fructose) and a non-reducing sugar (sucrose) at a range of concentrations $(0-15 \mathrm{~g} / 100 \mathrm{~g})$ under 2 bar pressure and a rotation of $1 \mathrm{rpm}$ using a steam retort to modify the green bean sugar content. Aroma analysis was carried out after coffee roasting by 
72 Gas chromatography mass spectrometry (GC-MS) with headspace solid phase micro extraction (SPME).

73 Sensory analysis in aroma was performed to determine the largest proportion of Robusta or treated

74 Robusta that could be blended with Arabica without any perceived sensory differences and accelerated

75 shelf life testing performed to explain the impact on aroma stability during storage. 


\section{Materials and methods}

77

78

79

80

81

82

83

84

85

86

87

88

89

90

91

92

93

94

95

96

\subsection{Coffee Samples}

Robusta samples were single-origin washed green beans from Vietnam. High grade Arabica coffee samples (Type AA: cupping 93/100) were sourced from Aberdares, Mount Kenya. They were both supplied by Edgehill coffee UK. Green coffee beans were positioned into a Modulyo Freeze Dryer 131103/08 JM (Edwards, Crawley, UK) at $-40{ }^{\circ} \mathrm{C}$ for $72 \mathrm{~h}$ until they achieved a humidity less than $5 \%$ before treatment. Freeze dried Robusta green beans were soaked with varying concentrations of individual sugar solution (glucose, fructose and sucrose) (Sigma-Aldrich, Poole, UK) with concentrations of 0, 3, 6, 9, 12, and $15 \mathrm{~g} / 100 \mathrm{~g}$ for $30 \mathrm{~min}$ at $100{ }^{\circ} \mathrm{C}$ with 2 bar pressure and a rotation of $1 \mathrm{rpm}$ using a steam retort with four replicates each. Control samples were treated with water only. Moisture content after treatment was controlled as detailed in our previous work (Liu, Yang, Linforth, Fisk, \& Yang, 2018), in brief treated coffee was dried naturally and placed into a salt chamber with saturated salt solution for two weeks (moisture content $11.5 \% \pm 0.5 \%$ ). Measurement of water loss over time was conducted by weighing the coffee samples at every step.

All coffee samples (4 replicates each) were roasted in the same batch using a 10 sample tray convection oven (Mono Equipment, Swansea, UK) for $20 \mathrm{~min}$ at $200^{\circ} \mathrm{C}$ and, after cooling by air, were ground using a coffee grinder (KG 49, Delonghi, Australia). Ground coffee was stored in a sealed aluminium bag at $80{ }^{\circ} \mathrm{C}$ after sieving (sieve size $710 \mu \mathrm{m}$ Endecotts, Essex, UK).

\subsection{Coffee Samples for Storage Test}

Coffee was stored at 5,25 , and $35{ }^{\circ} \mathrm{C}$ in a laboratory oven (Sanyo, Loughborough, UK). The moisture content of all samples before storage were measured less than $2 \%$. Samples were removed after 2, 4 and 
976 weeks and stored at $-80{ }^{\circ} \mathrm{C}$ ( 4 replicate samples). Control samples were stored from the start of the trial

98 at $-80{ }^{\circ} \mathrm{C}$. For instrumental analysis, all samples were analysed together at the end of the storage test in 99 a randomised order.

\subsection{Gas Chromatograph Mass Spectrometry (GC-MS)}

$1011.5 \mathrm{~g}$ of samples were placed into GC headspace vials $(20 \mathrm{~mL}, 22.5 \mathrm{~mm} \times 75.5 \mathrm{~mm}$, Sigma-Aldrich, UK)

102 (four replicates). 3-Heptanone was used as internal standard (15 $\mu \mathrm{L}, 0.01 \%$ 3-Heptanone (Sigma, Saint 103 Louis, USA) in methanol (Laboratory reagent grade, Fisher Scientific, UK)) to calibrate for any 104 instrument drift.

105 Aroma sampling conditions were chosen according to Liu, Yang, Linforth, Fisk, \& Yang, (2018), where 106 optimal conditions for pre-equilibrium time and temperature, extraction and injection are reported. In 107 brief, analysis was conducted using a trace 1300 series Gas Chromatography coupled with the Single108 Quadrupole Mass Spectrometer (Thermo Fisher Scientific, Hemel Hempstead, UK). Samples were 109 incubated with shaking at $40{ }^{\circ} \mathrm{C}$ for $5 \mathrm{~min}$. A 50/30 $\mu \mathrm{m}$ DVB/CAR/PDMS SPME Fibre (Supelco, Sigma 110 Aldrich, UK) was used to extract volatile compounds from the headspace of each samples. The SPME 111 fibre was extracted for 5 min then thermally desorbed for 2 min at $200{ }^{\circ} \mathrm{C}$, splitless mode, constant carrier 112 pressure of $18 \mathrm{psi}$, and then separated by GC-MS.

113 The column was a $30 \mathrm{~m}$ length ZB-WAX capillary column ( $0.25 \mathrm{~mm}$ internal diameter and $1.00 \mu \mathrm{m}$ film 114 thickness, Phenomenex, Macclesfield, UK). The conditions were as follows: $40{ }^{\circ} \mathrm{C}$ for 5 min, ramped to $115180{ }^{\circ} \mathrm{C}$ at $3{ }^{\circ} \mathrm{C} / \mathrm{min}$, and then ramped to $240{ }^{\circ} \mathrm{C}$ at $8{ }^{\circ} \mathrm{C} / \mathrm{min}$, held for $2 \mathrm{~min}$. Full scan mode was used in 116 a mass range of $\mathrm{m} / \mathrm{z} 20$ to 300 . 
117 Volatile compounds were identified by comparison of each mass spectrum with either the spectra from

118 standard compounds or with spectra in reference libraries (NIST/EPA/NIH Mass Spectral Library, 119 version 2.0, Faircom Corporation, U.S.). The relative abundant of volatiles was calculated from GC peak 120 areas, by comparison with the peak area of the internal standard.All samples were analysed in one run in 121 randomised order.

\section{2.4 Measurement for Physical Properties}

123 Colour was determined for four replicates with a Hunter Lab (ColourQuest XE, HunterLab, US) to 124 produce lightness (L), a value, and $b$ value. Positive $a$ and $b$ represent red and yellow, negative $a$ and $b$ 125 represent green and blue respectively (Hunter Lab, 2008). The conditions of the experiment were as 126 follows: standard illumination: D65, colorimetric normal observer angle: $10^{\circ}$, ASTM E308 RSIN Mode, 127 LAV, 1.00 Port, UV Nominal. The readings were made by CIELAB system. The Hunter Lab was 128 standardized by using the light trap standard (serial no. CQX2614) and diagnostic tile (serial no. 129 CQX2614). Coffee powders (1g) were put into cuvettes (SARSTEDT AG \& Co. D-51588) and directly 130 placed to the measurement aperture to test $\mathrm{L}$, $\mathrm{a}$ and $\mathrm{b}$ value with three positions selected at random. The 131 total colour difference $(\Delta \mathrm{E}), \Delta \mathrm{E}$ also can be calculated by equation and represents the difference between 132 the treated samples and the Arabica control.

$\Delta \mathrm{E}=\left[(\Delta \mathrm{L})^{2}+(\Delta \mathrm{a})^{2}+(\Delta \mathrm{b})^{2}\right]^{1 / 2}$

\subsection{Sugar Analysis by Liquid Chromatography-Mass Spectrometry}

135 Coffee powder $(0.1 \mathrm{~g})$ was positioned in a $50 \mathrm{~mL}$ centrifuge tube with $15 \mathrm{~mL}$ of boiling water and 136 vortexed for $5 \mathrm{~min}$. Samples then were centrifuged at $1600 \mathrm{~g}$ for $10 \mathrm{~min}$ at ambient temperature. After centrifugation, the liquid phase was transferred into a new glass vial. The above processes was repeated 
138 three times. The mixture was cooled to room temperature and then filtered using a syringe filter $(0.45$

$139 \mu \mathrm{m}, 40$ hydrophilic nylon syringe filter, Millipore Corporation). The final extract was diluted with

140 methanol $(\mathrm{MeOH})(1: 1)$ prior to Liquid chromatography-mass spectrometry (LC/MS) analysis (the 141 method was modified from Caporaso, Whitworth, Grebby, \& Fisk, (2018) and Perrone, Donangelo, \& 142 Farah, (2008).

143 The LC equipment (1100 Series, Agilent) consisted of a degasser (G1322A, Agilent), a pump (G1312A, 144 Agilent), an auto-sampler (G1313A, Agilent). This LC system was interfaced with a Quattro Ultima mass 145 spectrometer (Micromass, UK Ltd.) fitted with an electrospray ion source. The Luna 5u NH2 100A 146 column $(250 \times 3.20 \mathrm{~mm}, 5 \mu \mathrm{m}$, Phenomenex $)$ was used to separate sucrose, glucose and fructose at room 147 temperature. Chromatographic separation was carried with an isocratic elution mobile phase of $80 \%$ 148 acetonitrile. The flow rate was set at $0.7 \mathrm{~mL} / \mathrm{min}$, the volume injected was $5 \mu \mathrm{L}$.

149 Peaks were determined by comparing retention times to those of standard compounds. Calibration curves 150 were made of sucrose, glucose and fructose standards (Sigma Aldrich®). Standards were prepared at 151 concentration of $1,2.5,5,7.5$, and $10 \mathrm{mg} / \mathrm{mL}$ in 50:50 MeOH: $\mathrm{H}_{2} \mathrm{O}$. The respective peak areas were used 152 for the quantification.

$153 \quad 2.6$ Sensory Evaluation

154 Robusta samples treated by soaking in $15 \mathrm{~g} / 100 \mathrm{~g}$ fructose $(15 \mathrm{~F})$ were selected to be tested in the sensory 155 study. The coffee brew for sensory evaluation were freshly brewed in a cafetière just before the test start 156 to avoid any flavour loss and oxidation. According manufacturers' instruction, $54 \mathrm{~g}$ of coffee was 157 weighed and add in the 8-cup capacity cafetière (Argos, Stafford, UK). $860 \mathrm{~mL}$ boiling water was then 158 poured into the cafetière with 5 times stir. The coffee were then wait for 3 min before depressing the 
159 plunger. Brewed coffee $(10 \mathrm{~mL})$ was then poured into amber glass vessels and cooled down to room 160 temperature $\left(20 \pm 2{ }^{\circ} \mathrm{C}\right)$ for sniffing test.

161 This study was approved by School of Bioscience Ethic Committee at the University of Nottingham 162 (SBREC160138A), a small incentive was provided to participants. All sensory tests were conducted 163 under northern hemisphere lighting at the Sensory Science Centre of the University of Nottingham in the 164 individual sensory booths. Ninety-eight volunteers were recruited from students and staff at University 165 of Nottingham, all participants have signed informed consent. Participants were invited for one session 166 which lasted approximately $30 \mathrm{~min}$, in the session, a total of 7 triangle tests were carried out. The 167 objective of the sensory test was to determine the similarity between non-treated Robusta and Arabica 168 and the blended Arabica with Robusta (treated or control). In previous studies we have shown that 169 participants can perceive when a minimum of $40 \%$ of Robusta is blended with Arabica (Liu, Yang, 170 Linforth, Fisk, \& Yang, 2018). Therefore, in this experiment, a blending ratio of $20 \%$ and $40 \%$ Robusta 171 with Arabica were compared with 100\% Arabica to confirm this finding. For fructose-treated Robusta, samples with 20\%, 40\%, 60\% and 80\% blending with Arabica were used to compare with 100\% Arabica.

173 For each triangle test, three samples were given to the volunteers, and they were instructed to smell the 174 samples from left to right and select the odd one. A two minute break was given between triangles tests. 175 No other prior knowledge or training was given to the assessors. A randomised sampling order was used 176 between and within each triangle test.

\section{$177 \quad 2.7$ Statistical Analysis}

178 Experiments were carried out in quadruplicate. Data is presented as a mean value with standard deviation 179 and samples were compared by analysis of variance (ANOVA) using samples as the fixed effect and 
180 followed by Tukey's HSD post-hoc test, $\mathrm{p}<0.05$ was regarded as significant. All statistical analyses 181 were conducted using either IBM $^{\circledR}$ SPSS $^{\circledR}$ Statistics version 21.0.0 or Excel XLSTAT (Version 182 2015.5.01.23373). All sensory data was collected and analysed using Compusense Cloud (Compusense, 183 Ontario, Canada). Number of responses was compared to the critical tables in BS EN ISO 4120: 2007 $184(\alpha=0.05$ for difference testing; $\alpha=0.2, \beta=0.05, \mathrm{pD}=30 \%$ for similarity testing). 


\section{Results and discussion}

\subsection{Impact of Treatment on Sugar Content and Bean Colour after Roasting}

187 The sugar content in the green coffee beans and the colour of the roasted coffee beans are presented in 188 Table 1. Non-treated Robusta had significantly lower concentrations of sucrose when compared with 189 Arabica (respectively: $3.20 \mathrm{~g} / 100 \mathrm{~g} \pm 0.38 ; 6.20 \mathrm{~g} / 100 \mathrm{~g} \pm 0.10)(\mathrm{p}<0.05)$. There was no significant 190 difference in the glucose concentration between Arabica and non-treated Robusta $(\mathrm{p} \geq 0.05)$. However, the fructose concentration in the non-treated Robusta $(0.76 \mathrm{~g} / 100 \mathrm{~g} \pm 0.20)$ was significantly higher than 192 Arabica $(0.13 \mathrm{~g} / 100 \mathrm{~g} \pm 0.06)$.

193 To accelerate the diffusion of sucrose, glucose and fructose into the coffee beans, pre-soaking was carried out at 2 bar pressure. A rotation of $1 \mathrm{rpm}$ was used to create even distribution of the treatment solution.

The process control (water treated Robusta) was significantly lower in sucrose, glucose and fructose content when compared with the non-treated Robusta. This is due to the nature of the treatment process as, sucrose, glucose and fructose are water soluble and can be leached out into the process water during 198 the treatment.

Increasing the sugar concentration in the treatment solution increased the sugar content in the treated green beans (Table 1). At the highest treatment level, Robusta samples were treated by soaking in 15 $\mathrm{g} / 100 \mathrm{~g}$ of individual sugars (fructose, glucose, and sucrose), which are represented as 15F, $15 \mathrm{G}$ and $15 \mathrm{~S}$ accordingly. There was $4.98 \mathrm{~g} / 100 \mathrm{~g}$ sucrose in the $15 \mathrm{~S}$ treated green beans; $7.39 \mathrm{~g} / 100 \mathrm{~g}$ glucose in the $15 \mathrm{G}$ treated green bean; $7.35 \mathrm{~g} / 100 \mathrm{~g} 15 \mathrm{~F}$ in the fructose treated green bean. At the highest sucrose treatment level the treated Robusta coffee still had a lower sucrose concentration $(4.98 \mathrm{~g} / 100 \mathrm{~g})$ than Arabica $(6.20 \mathrm{~g} / 100 \mathrm{~g})$. There was a significant increase in glucose and fructose concentrations between 
the glucose and fructose treated Robusta samples compared with the Arabica sample (Table 1). It should

207 be noted that less sucrose was detected in the sucrose treated samples than glucose or fructose in their

208 treated samples. Sucrose is a disaccharide with the molecular weight $342 \mathrm{~g} / \mathrm{mol}$ and may penetrate the 209 sample matrix less readily than monosaccharides such as glucose $(180 \mathrm{~g} / \mathrm{mol})$ and fructose $(180 \mathrm{~g} / \mathrm{mol})$.

210 Colour analysis of the coffee bean samples showed significant differences in $\mathrm{L}, \mathrm{a}, \mathrm{b}(\mathrm{p}<0.05)$ between 211 Arabica beans and the non-treated Robusta. $\Delta \mathrm{E}$ was used to determine the overall distance between two 212 colours. According to the previous study, $\Delta \mathrm{E}$ of 3.0 is the minimum colour difference that human eyes 213 can detect (depends on the hue) (Martínez-Cervera, Salvador, Muguerza, Moulay, \& Fiszman, 2011).

214 Clear differences were seen between the Arabica and the non-treated Robusta with a total colour 215 difference $\Delta \mathrm{E}$ of 7.48 (Table 1). This is the greatest colour difference between the Arabica and all coffee 216 samples. At $15 \mathrm{~S}$ treatment, $12 \mathrm{G}$ and $15 \mathrm{G}$ treatment and $9 \mathrm{~F}, 12 \mathrm{~F}$ and $15 \mathrm{~F}$ treatment, total colour 217 differences were lower than 3, and were the least colour difference when compared with Arabica. As a 218 result, it can be seen that sugar pre-treatment reduced the colour difference between Arabica and Robusta 219 after roasting.

Increasing the levels of flavour precursors (sucrose, glucose and fructose) in the Robusta beans did alter 221 the colour of the beans making the treated coffee more similar to that of the Arabica bean. The colour 222 formation is mainly due to the Maillard reaction (Bastos, 2012) and sugar caramelization processes, 223 which can occur simultaneously, hence it is hard to separate the two reactions (Wong, Abdul Aziz, \& 224 Mohamed, 2008). It should be noted that the reducing sugars (glucose and fructose) had a greater impact 225 on the colour change than the non-reducing sugar sucrose suggests that both Maillard reaction and caramelization are of importance. Ganesan and Benjakul did a similar study on the basis of glucose treatment on pidan white (pickled duck eggs). They hypothesised and proved that adding Maillard 
228 chemistry precursors (glucose) could improve brown colour development principally through 229 accelerating the Maillard reaction (Ganesan, Benjakul, \& Baharin, 2014), which consistent with our 230 result in table 1.

\subsection{Determination of the Volatile Compounds in Coffee after Treatment}

232 Thirty-four volatile compounds were identified in all coffee samples, they was screened and selected as compounds that have previously been shown to be key aroma compounds with sensory significance in coffee. These aroma compounds are shown in table 2 and include 5 furans, 2 organic acids, 5 heterocyclic compounds (N containing), 4 sulphur-containing compounds, 2 aldehydes, 3 ketones and 9 pyrazines, 1 ether, 1 alcohol and 2 phenolic compounds. Their linear retention index, identification method and related odour description are illustrated in Table 2.

\subsection{Summary of All Coffee Samples via Volatile Chemistry}

Principal component analysis (PCA) was used to illustrate the variation in the level of the 34 volatiles compounds formed during the roasting process (Figure 1). The first principal component (PC1) represents $63.9 \%$ of the variance in the whole dataset and was negatively correlated with pyrazines and phenolic compounds and positively correlated with furans, ketones, aldehydes, ether, alcohol and acids on the right. The second principal component (PC2) represents $18.6 \%$ of the variance and has a positive correlation with pyrroles and negative correlation with sulphur-containing compounds. The non-treated Robusta sample had greater levels of pyrazines and phenolic compounds (left with triangle mark). While Arabica have a positive correlation with acids, furans, ketones and aldehydes (right with triangle mark). The main categories of compounds found at a higher proportion in Arabica were furans, acids, aldehydes and pyridines, which literature suggests are related to the aroma of roasted sweet caramel (Petisca, Pérez- 
249 Palacios, Farah, Pinho, \& Ferreira, 2013). Robusta on the other hand is known to have a spicy burnt 250 earthy odour due to higher concentrations of pyrazines and derivatives (Kerler, 2010), which is 251 concordant with our results in the Figure 1. Increasing the levels of flavour precursors (sucrose, fructose 252 and glucose)moved the aroma profile from left to right, closer to Arabica. The 15F treated coffees (square marked in the figure 1) was the closest to the Arabica samples.

254 The extent of the change in aroma profile was more marked for the reducing sugars (glucose and fructose) when compared to the non-reducing sugar (sucrose) suggestions that whilst caramelisation may be important, Maillard chemistry is the major drives factor in the change in aroma profile and is critically important for binding the gap between Arabica and Robusta.

\subsection{Aroma Chemistry}

259

260

261

262

263

264

265

266

267

268

269

The aroma profile for Arabica, treated and non-treated Robusta sample is illustrated in Figure 2, where the level of 34 key volatile compounds in treated and non-treated Robusta coffee are normalised by their respective concentrations in Arabica coffee (100\%). Significant differences were shown in all 34 key aroma compounds between Arabica and Robusta (Figure 2 (a)). Robusta coffee had 2 to 4 times higher concentration of all pyrazines, pyrroles, phenolic compounds and 4-Methylthiazole when compare with Arabica coffee. However, for the rest of the volatile compounds, such as furans, ketones, aldehydes, and acids, non- treated Robusta coffee had up to 8 times lower concentration than Arabica coffee.

As shown in figure 2 (b), the aroma profile for the process control Robusta sample indicated significant differences $(\mathrm{p}<0.001)$ in 32 volatile compounds compared to Arabica apart from pyrrole and disufide dimethyl. These include a significantly greater level of pyrazines, phenolic compounds and 4methylthiazole and lower levels of compounds such as furans, ketones, acids and aldehydes. Similar to 
non-treated Robusta, the process control Robusta had a similar pattern but the differences were smaller.

271 These included a significantly decreased levels of compounds such as pyrazines, furans, aldehydes,

272 ketones and pyrroles. This change can be explained by the leaching of water soluble precursors during treatment process as shown in table 1. Volatiles such as furfural, 2-methylfuran have been reported as sugar degradation products that can be affected in this way (Flament, 2002). In addition, an alteration to the bean density (from $0.75 \mathrm{~g} / \mathrm{mL}$ to $0.62 \mathrm{~g} / \mathrm{mL}$ ) could also alter the thermal reaction pathways during aroma formation. High density beans are more resistant to absorption of heat and takes a longer time to roast (Pittia, Dalla Rosa, \& Lerici, 2001). Applying steam and pressure to the beans may open up bean pores and could modify the density of the green coffee beans. As a result, treated beans could have a lower density and be less resistant to heat.

Figure 2 (c) indicated the aroma profile between Arabica and $15 \mathrm{~F}$ treated Robusta. There were no significant differences in the concentration of 16 compounds (including all pyrazines, aldehydes, 2, 5dimethylfuran, 4-methylthiazole, 4-vinylguaiacol, 1-ethylpyrrole and 2, 5-dimethylpyrrole) between Arabica and $15 \mathrm{~F}$ treated Robusta. Although most furans, ketones and organic acids were still lower in the $15 \mathrm{~F}$ treated Robusta coffee compared with the Arabica, all furans, ketones and organic acids indicated 285 a significant increase in $15 \mathrm{~F}$ treated Robusta (2-3 fold) when compare with non-treated Robusta and processing controlled Robusta, which made it closer to Arabica's profile.

Figure 2 (d) indicated the aroma profile between Arabica and 15G treated Robusta. There were no significant differences in 6 compounds (including 2, 5-dimethylpyrazine, 2-ethyl-5-methylpyrazine, methylpyrazine, pyrazine, 1-ethylpyrrole, and 2, 5-dimethylfuran) between Arabica coffee and 15G treated Robusta coffee. Some pyrazines (2, 5-Dimethylpyrazine, 2-ethyl-5-methylpyrazine, methyl pyrazine, pyrazine) indicated a significant decrease in 15G treated Robusta (60\% - 100\%) compared with 
non-treated Robusta (Figure 2 (a)). The concentration of 1-ethylpyrrole and 2, 5-dimetylfuran increased around $30 \%$ to $50 \%$ respectively in the $15 \mathrm{G}$ treated Robusta when compared with the non-treated one.

Figure 2 (e) shows the aroma profile between Arabica and 15S treated Robusta. There were no significant difference in the concentration of 7 compounds (2, 5-dimethylpyrazine, 2-ethyl-5-methylpyrazine, methylpyrazine, pyrazine, 1-ethylpyrrole, 2, 5-dimethylfuran and furfural) between Arabica coffee and 15S treated Robusta coffee. Both glucose treated Robusta (15G) and sucrose treated Robusta (15S) had a similar pattern, apart from the relative concentration of furfural, which showed a significant increase in $15 \mathrm{~S}$ treated Robusta (26\%) compared with $15 \mathrm{G}$ treated Robusta sample.

The significant rise in the ketone, furan and acid compounds in the sugar treated Robusta may due to the formation of those compounds through carbohydrate pyrolysis and sugar degradation (Flament, 2002). Research has revealed that sugar decomposition enhances the volatilization and formation of formic acid, acetic acid and lactic acid in the initial stages of roasting (Yeretzian, Jordan, Badoud, \& Lindinger, 2002). In the later stages, during roasting at high temperature, furaneol and hydroxymethylfurfural are generated via sugar caramelization. However, aroma formation is more likely through the Maillard route than caramelization due to lower activation energy in the presence of reactive nitrogen species (amino acids) (Hodge, 1953; Yeretzian, Jordan, Badoud, \& Lindinger, 2002). The formations of these furans is thought to be greatly dependent on the sugar content (Nie et al, 2013). The sugar treatment level could therefore affect the formation of furans. Pyrazine is known to be predominant in Robusta and is formed by amino acids and reducing sugars following the Maillard reaction (Ehiling et al 2005). Koehler, and Odell 1970, discovered that increasing ( 3 fold) the amounts of sugar added could decrease the concentration of pyrazines generated, and the assumption was that excess sugar affected the reactant ratio hence 
313 decreasing pyrazine levels. That could also be the reason for the lower pyrazine levels observed in sugar treated Robusta.

315 Pyrroles and pyridines were significantly decreased (around 2 fold) in the sugar treated Robusta (Figure

3162 (c), (d), (e)). These two groups of compounds are formed as a result of the thermal degradation of

317 Amadori intermediates. The intermediate products can either cyclize to form these nitrogenous 318 heterocyclic compounds, or go to a different route where cleavage and formation of rearranged sugars 319 occur. Due to the rearranged sugars comprising of the intact chain of the starting sugar and the original 320 amine that was liberated, less or different volatile aroma compound were created (Jousse, Jongen, 321 Agterof, Russell, \& Braat, 2002). Moreover, pyrroles and pyridines have also been reported as pyrolysis 322 products of trigonelline (Flament, 2002). The reduced pyrroles and pyridines relative concentration may 323 be therefore due to the trigonelline leaching out during the pre-treatment process, which is confirmed by 324 the process control (Figure $2(\mathrm{~b}))$.

325 Of the three different sugars used to treat Robusta samples (15F, 15S and 15G), 15F treated Robusta 326 sample was found to be the optimum treatment conditions with the most compounds showing no 327 significant difference compare with Arabica. It indicated that the formation of the volatile compounds 328 can be affected by the types of sugar involved in the Maillard reaction and caramelization during the 329 roasting process, as also reported by Brands \& Van Boekel, 2001. Reducing sugar both glucose and 330 fructose (monosaccharides) were more reactive than the non-reducing sugar sucrose (disaccharides) (Van 331 Boekel \& Brands, 2005).

332 For monosaccharides, ketoses such as fructose give rise to the corresponding Heyns compound, whilst 333 the Aldoses such as glucose give rise to the Amadori intermediate compounds (Brands \& Van Boekel, 334 2001). There are conflicting reports in the literature regarding the issue of reactivity of sugars, several 
studies (Spark, 1969; Baxter, 1995) support that glucose is more reactive, while other researches claim that fructose is more reactive (Kato, Yamamoto, \& Fujimaki, 1969; Mauron, 1981; Suarez, Etlinger, Maturana, \& Weitman, 1995; Walton, McPherson, \& Shilton, 1989). Further studies indicated that the relative reaction rates vary for both glucose and fructose depending on the reaction conditions (Brands \& Van Boekel, 2001; Laroque, Inisan, Berger, Vouland, Dufossé, \& Guérard, 2008; Rewicki, Kersten, Helak, Nittka, \& Tressl, 2005).

In our study, 15F treated Robusta generated more furans, ketones, aldehydes and acetic acid compared with $15 \mathrm{G}$ treated Robusta, which agreed with the study on the flavour precursors in the Maillard reaction done by Kraehenbuehl et al. 2010. On the other hand, formation of pyrazines significantly decreased in 15F treated Robusta compared with $15 \mathrm{G}$ treated Robusta. No significant difference in pyrazines can be observed in the $15 \mathrm{~F}$ treated Robusta compared with Arabica. As discussed above, only $15 \mathrm{~F}$ treated Robusta samples were used for the sensory evaluation.

\subsection{Influence of Accelerated Shelf-life Storage on the Volatile Compounds}

The relative change (percentage) in aroma of the three coffee samples stored for six weeks at $35{ }^{\circ} \mathrm{C}$ is shown in figure 3 . The relative aroma difference during storage was normalised to $100 \%$ of its original level in each coffee. The use of relative abundance in figure 3 was used to avoid different starting points for Arabica, Robusta and treated Robusta coffee before storage as these two varieties might contain different amounts of the volatile compounds after roasting.

For Arabica, all compounds significantly decreased over the storage period between $25 \%-60 \%$ (p< 0.05). The only exception was acids that increased around two fold over the six weeks' time. The concentrations of total pyrroles, pyrazines, aldehydes, furans reduced significantly during six week 
storage at $35{ }^{\circ} \mathrm{C}$ in Arabica, non-treated Robusta and 15F treated Robusta. Non-treated Robusta, treated

357 Robusta and Arabica all showed no significant difference in the ketones after six weeks stored at $35^{\circ} \mathrm{C}$

358 when compared with the control.

359 The aroma of $15 \mathrm{~F}$ treated Robusta was more stable during 6 weeks storage compared with Arabica, as 360 most of the volatiles in Arabica coffee showed a greater loss over storage when compared to the treated 361 Robusta. The only exception was that 15F treated Robusta generated 35\% more acids (include acetic acid and propanoic acid) compared with Arabica during the six weeks stored. The formation of acetic acid can be due to degradation of small to medium chained carbohydrates such as glucose, sucrose and fructose (Illy \& Viani, 2005). The higher fructose content may result in a greater acid release in the roasted coffee (Farah, 2012; Rewicki, Kersten, Helak, Nittka, \& Tressl, 2005). Moreover, previous studies on staling and rancidity in coffee concluded that the volatile compounds (such as furfural and acetaldehyde) can be oxidised to the corresponding volatile acids during coffee storage period (Elder, 1937). $15 \mathrm{~F}$ treated Robusta coffee generated around $25 \%$ more furfural compared with Arabica (Figure

2 (c)). Therefore, higher volatile acids formation during coffee storage could also be explained by the oxidation of aroma constituents. Whilst the difference in stability of aroma compounds in the Arabica compared to the Robusta and treated Robusta cannot be clearly explained, it may be due to the present

372 of different levels of micro nutrients, different volatiles and different bean chemistry. However, it is clear 373 that the aroma of Robusta and treated Robusta were more stable. This was especially evident for 374 pyrazines, aldehydes and furans.

\subsection{Sensory evaluation}

376 Fructose treated Robusta coffee $(15 \mathrm{~F})$ was blended with up to $80 \%$ Arabica coffee and compared with 377 the Arabica control to identify the maximum blend ratio without a perceive aroma difference. The results 
378 for the numbers of correct responses in a sensory triangle test evaluation of brewed coffee are shown in 379 Table 3. According to ISO4120:2007, samples were classed as being similar to Arabica if the number of 380 correct responses was less than 40 out of 98.

381 In agreement with Liu, Yang, Linforth, Fisk, \& Yang (2018), participants could not tell a difference 382 between Arabica and Arabica containing 20\% Robusta blend, but once the blending ratio increased to $38340 \%$ Robusta, participants could tell that the aroma was significantly different from the $100 \%$ Arabica 384 sample. Interestingly, when comparing Arabica with $15 \mathrm{~F}$ treated Robusta blended with Arabica, 385 participants could not discriminate between the aroma of the two samples, no matter the percentage of 386 the blending (from $20 \%$ to $80 \%$ blends). The sensory evaluation results are consistent with the volatile 387 analysis which showed that the $15 \mathrm{~F}$ treated samples were the most similar to Arabica, and enable 388 therefore on an aroma basis an increase in blending ratio from $20 \%$ Robusta $80 \%$ Arabica to $80 \%$ treated 389 Robusta 20\% Arabica . 


\section{Conclusions}

391 In conclusion, this project has successfully developed a model system for the evaluation of flavour 392 precursors in green beans and proposed how modifying green bean carbohydrate profile can result in an 393 enhanced aroma profile where the aroma of Robusta coffee is more similar to Arabica. Analytical results 394 indicated that the inclusion of fructose resulted in the most similar aroma profile to Arabica. Sensory test 395 results validated this finding, which proved that $15 \mathrm{~F}$ treated Robusta had a similar perceived aroma as 396 Arabica. The maximum permissible blending proportion of Robusta increased from $20 \%$ for the non397 treated Robusta coffee to $80 \%$ for the $15 \mathrm{~F}$ treated Robusta coffee. It is clear from these findings that 398 modification of the aroma precursors (especially fructose addition) changes the roasted coffee aroma 399 profile and enables a higher Robusta blending ratio. Furthermore, the aroma stability of the treated 400 Robusta significantly increased.

\section{Acknowledgment}

402 This work was supported by the Biotechnology and Biological Sciences Research Council, United 403 Kingdom [grant number BB/R01325X/1].

404 We acknowledge Lim Mui, Vlad Dinu, Helen Allen and Steven Johnson from the University of 405 Nottingham for their help and support with technical issues. The authors thank Deepa Agarwal and 406 Nicola Caporaso for proofreading the paper. 
407

408

409

410

411

412

413

414

415

416

417

418

419

420

421

422

423

424

425

426

427

428

\section{References}

Akiyama, M., Murakami, K., Ikeda, M., Iwatsuki, K., Wada, A., Tokuno, K., Onishi, M., \& Iwabuchi, H. (2007). Analysis of the headspace volatiles of freshly brewed arabica coffee using solid-phase microextraction. Journal of Food Science, 72, 388-396.

Bastos, D. M., Monaro, É., Siguemoto, É., Séfora, M. (2012). Maillard Reaction Products in Processed Food: Pros and Cons. Food Industrial Processes - Methods and Equipment, 10, 5772.

Baxter, J. H. (1995). Free Amino Acid Stability in Reducing Sugar Systems. Journal of Food Science, $60,405-408$.

Blank I., Sen A., \& Grosch, W. (1991). Aroma impact compounds of arabica and robusta coffee. Qualitative and quantitative investigations. Colloque Scientifique International sur le Cafe, 117129.

Boothroyd, E., Linforth, R. S. T., Jack, F., \& Cook, D. J. (2014). Origins of the perceived nutty character of new-make malt whisky spirit. Journal of the Institute of Brewing, 120, 16-22.

Brands, C. M. J., \& Van Boekel, M. A. J. S. (2001). Reactions of Monosaccharides during Heating of Sugar-Casein Systems: Building of a Reaction Network Model. Journal of Agricultural and Food Chemistry, 49, 4667-4675.

Caporaso, N., Whitworth, M. B., Grebby, S., \& Fisk, I. D. (2018). Non-destructive analysis of sucrose, caffeine and trigonelline on single green coffee beans by hyperspectral imaging. Food Research International, 106, 193-203.

Elder, L. W. (1937). Staling vs. Rancidity in Roasted Coffee - Oxygen Absorption by the Fat Fraction. Industrial and Engineering Chemistry, 29, 267-269.

Farah, A. (2012). Coffee Constituents. Coffee, 21-58. 
429 Fisk, I. D., Kettle, A., Hofmeister, S., Virdie, A., \& Kenny, J. S. (2012). Discrimination of roast and ground coffee aroma. Flavour, 1, 14.

Flament, (2002). Coffee flavor chemistry. West Sussex.

432 Ganesan, P., Benjakul, S., \& Baharin, B. S. (2014). Maillard Reaction of Pidan White as Inhibited by Chinese Black Tea Extract (Camellia sinensis) in the Pickling Solution. Korean Journal for Food Science of Animal Resources, 34, 403-407.

International Coffee Organization. The current state of the global coffee trade |CoffeeTradeStats. (2016). http://www.ico.org/monthly_coffee_trade_stats.asp Accessed 01/08/18.

Illy, A., \& Viani, R. (2005). Espresso Coffee: The Science of Quality: Elsevier Academic.

ISO, (2007). International Organization for Standardization (ISO). Sensory Analysis-MethodologyTriangle Test, BS ISO 4120. BSI, London, UK.

Jousse, F., Jongen, T., Agterof, W., Russell, S., \& Braat, P. (2002). Simplified Kinetic Scheme of Flavor Formation by the Maillard Reaction. Journal of Food Science, 67, 2534-2542.

Kato, H., Yamamoto, M., \& Fujimaki, M. (1969). Mechanisms of Browning Degradation of d-Fructose in Special Comparison with d-Glucose-Glycine Reaction. Agricultural and Biological Chemistry, 33, 939-948. reactions. Journal of Agricultural and Food Chemistry, 18, 895. Precursors in the Maillard Reaction.

450

Kerler, J., Winkel, C., Davidek, T. and Blank, I. (2010). Wiley-Blackwell, Oxford, UK.

Koehler, P. E., G. V. Odell. (1970). Factor affecting the formation of pyrazine compounds in sugar-amine

Kraehenbuehl, K., Davidek, T., Devaud, S., \& Mauroux, O. (2010). Basic and Acidic Sugars as Flavour

Laroque, D., Inisan, C., Berger, C., Vouland, É., Dufossé, L., \& Guérard, F. (2008). Kinetic study on the Maillard reaction. Consideration of sugar reactivity. Food Chemistry, 111(4), 1032-1042. 
452 Liu, C., Yang, Q., Linforth, R., Fisk, I. D., \& Yang, N. (2018). Modifying Robusta Coffee Aroma by Green Bean Chemical Pre-Treatment. Food Chemistry.

454 Martínez-Cervera, S., Salvador, A., Muguerza, B., Moulay, L., \& Fiszman, S. M. (2011). Cocoa fibre 455 and its application as a fat replacer in chocolate muffins. LWT - Food Science and Technology, $44,729-736$.

Mauron, J. (1981). The Maillard reaction in food; a critical review from the nutritional standpoint. Progress in Food and Nutrion Science, 5, 5-35.

Mottram, R. The LRI \& Odour Database. (2018). http://www.odour.org.uk/ Accessed 27/06/18.

Nie, S., Huang, J., Hu, J., Zhang, Y., Wang, S., Li, C., Marcone, M., \& Xie, M. (2013). Effect of pH,

Perrone, D., Donangelo, C. M., \& Farah, A. (2008). Fast simultaneous analysis of caffeine, trigonelline, 464 nicotinic acid and sucrose in coffee by liquid chromatography-mass spectrometry. Food 465 Chemistry, 110, 1030-1035.

Petisca, C., Pérez-Palacios, T., Farah, A., Pinho, O., \& Ferreira, I. M. P. L. V. O. (2013). Furans and other volatile compounds in ground roasted and espresso coffee using headspace solid-phase microextraction: Effect of roasting speed. Food and Bioproducts Processing, 91, 233-241.

Piccino, S., Boulanger, R., Descroix, F., \& Shum Cheong Sing, A. (2014). Aromatic composition and potent odorants of the "specialty coffee" brew "Bourbon Pointu" correlated to its three trade classifications. Food Research International, 61, 264-271.

Pittia, P., Dalla Rosa, M., \& Lerici, C. R. (2001). Textural Changes of Coffee Beans as Affected by Roasting Conditions. LWT - Food Science and Technology, 34, 168-175. 
474 Rewicki, D., Kersten, E., Helak, B., Nittka, C., \& Tressl, R. (2005). Mechanistic Studies on the Formation of Maillard Products from [1-13C]-D-Fructose. Maillard Reactions in Chemistry, Food and Health, 61-68.

Semmelroch, P., \& Grosch, W. (1996). Studies on Character Impact Odorants of Coffee Brews. Journal of Agricultural and Food Chemistry, 44, 537-543.

Spark, A. (1969). Role of amino acids in non-enzymic browning. Journal of the Science of Food and Agriculture, 20, 308-316.

Suarez, G., Etlinger, J. D., Maturana, J., \& Weitman, D. (1995). Fructated protein is more resistant to fluorophores. Archives of Biochemistry and Biophysics, 321, 209-213.

Van Boekel, M. A. J. S., \& Brands, C. M. J. (2005). Heating of Sugar-Casein Solutions: Isomerization and Maillard Reactions. The Maillard Reaction in Foods and Medicine, 154-159.

Walton, D. J., McPherson, J. D., \& Shilton, B. H. (1989). Fructose mediated crosslinking of proteins. Progress in Clinical and Biological Research, 304, 163-170.

Wong, K. H., Abdul Aziz, S., \& Mohamed, S. (2008). Sensory aroma from Maillard reaction of individual and combinations of amino acids with glucose in acidic conditions. International Journal of Food Science and Technology, 43, 1512-1519.

Wrigley, G. (1988). Coffee: Longman Scientific and Technical.

Yeretzian, C., Jordan, A., Badoud, R., \& Lindinger, W. (2002). From the green bean to the cup of coffee: investigating coffee roasting by on-line monitoring of volatiles. European Food Research and Technology, 214(2), 92-104.

Hodge, J. E. (1953). Dehydrated Foods, Chemistry of Browning Reactions in Model Systems. Journal of Agricultural and Food Chemistry, 1(15), 928-943. 
